\title{
Coupled-mode analysis of light propagation in optically active crystals
}

\author{
Amnon Yariv \\ Department of Electrical Engineering, California Institute of Technology, Pasadena, California 91109
}

James F. Lotspeich

Hughes Research Laboratories, 3011 Malibu Canyon Road, Malibu, California 90265

Received August 3, 1981

\begin{abstract}
A new mathematical formalism, based on a coupled-mode approach, is presented to describe light propagation in birefringent optically active crystals. An arbitrary light wave in the medium is represented as an expansion in terms of linearly polarized eigenmodes; explicit solutions of the equations for the coupled-wave amplitudes show how power is transferred between the principal components in the presence of optical activity. The (generally elliptically polarized) eigenmodes of the optically active crystal are also derived by a diagonalization of the coupledmode equations. The analysis is extended to include electro-optic crystals, yielding a more general, complex coupling parameter. The predictions of the theory are in excellent agreement with spectral-transmission measurements in $\mathrm{AgGaS}_{2}$ near its isoindex point at $497 \mathrm{~nm}$.
\end{abstract}

\section{INTRODUCTION}

The propagation of light in optically active crystals, which may also be birefringent, is a topic of continuing theoretical and experimental interest since many crystals that are potentially useful in device applications are optically active. Among these crystals are quartz (in which the effect was discovered), silver gallium sulfide $\left(\mathrm{AgGaS}_{2}\right)$, and sodium chlorate. The problem of light propagation in such crystals has been analyzed by Szivessy, ${ }^{1}$ Born, ${ }^{2}$ and others and reviewed by Condon, ${ }^{3}$ Ramachandran and Ramaseshan, ${ }^{4}$ and Mathieu. ${ }^{5}$ The mathematical treatment is sufficiently complex that $\mathrm{Nye}^{6}$ found it necessary to ".. warn the reader that the calculation contains some subtleties which have led to errors in some of the published work."

We present a new analytical approach to treating wave propagation in birefringent (e.g., uniaxial), optically active crystals. The approach is based on the coupled-mode formalism ${ }^{7}$ initially developed for guided-wave optics. The working formulas resulting from this point of view and the physical insight that they afford make the application of the results to experimental situations straightforward. Since many of the optically active crystals are electro-optic as well, we also apply the coupled-mode formalism to the problem of propagation in optically active electro-optic crystals. We conclude by comparing some of the predictions of the theory with experimental measurements in $\mathrm{AgGaS}_{2}$.

\section{COUPLED-MODE FORMULATION}

Consider the general problem of a light wave propagating along an arbitrary direction $\zeta$ inside a birefringent optically active crystal. We can use the two linearly (and orthogonally) polarized plane-wave eigenmodes for light propagation in a birefringent crystal as a basis set for expanding the field in the presence of optical activity.
For wave propagation along the $\zeta$ direction in a uniaxial crystal, the two eigenmodes have their $\mathbf{D}$ vectors along the $\eta$ direction (extraordinary) and the $\xi$ direction (ordinary), as shown in Fig. 1. The magnitudes of the wave-propagation constants are

$$
k_{\eta}=\frac{\omega}{c} n_{\eta}
$$

and

$$
k_{\xi}=\frac{\omega}{c} n_{\xi},
$$

where

$$
\begin{aligned}
n_{\eta}{ }^{-2} & =n_{e}{ }^{-2} \cos ^{2} \theta+n_{0}{ }^{-2} \sin ^{2} \theta, \\
n_{\xi} & =n_{0} .
\end{aligned}
$$

The $x-y-z$-axis system in Fig. 1 represents the crystallographic principal axes. In the $\xi-\eta-\zeta$ system, the transverse dielectric tensor is diagonal with elements $\varepsilon_{\xi \xi}=\varepsilon_{0} n_{0}^{2}$ and $\varepsilon_{\eta \eta}$ $=\varepsilon_{0} n_{\eta}^{2}$.

The effect of optical activity is to introduce off-diagonal elements into the susceptibility tensor so that the relationship between the transverse polarization and field becomes

$$
\left|\begin{array}{c}
P_{\xi} \\
P_{\eta}
\end{array}\right|=\varepsilon_{0}\left|\begin{array}{cc}
n_{\xi}^{2}-1 & i a \\
-i a & n_{\eta}^{2}-1
\end{array}\right|\left|\begin{array}{c}
\mathscr{E}_{\xi} \\
\mathscr{E}_{\eta}
\end{array}\right|
$$

The real constant $a$ accounts for the optical activity.

We take the total complex transverse field as

$$
\begin{aligned}
\mathbf{E}(\xi, \eta, \zeta)=\hat{\xi} \mathscr{E}_{\xi}(\zeta) \exp & {\left[i\left(\omega t-k_{\xi} \zeta\right)\right] } \\
& +\hat{\eta} \mathscr{E}_{\eta}(\zeta) \exp \left[i\left(\omega t-k_{\eta} \zeta\right)\right]+\text { c.c. }
\end{aligned}
$$

In the absence of optical activity ( $a=0$ ), the field [Eq. (2)] with amplitudes $\mathscr{E}_{\xi}$ and $\mathscr{E}_{\eta}$ independent of $\zeta$ is a solution of the wave equation 


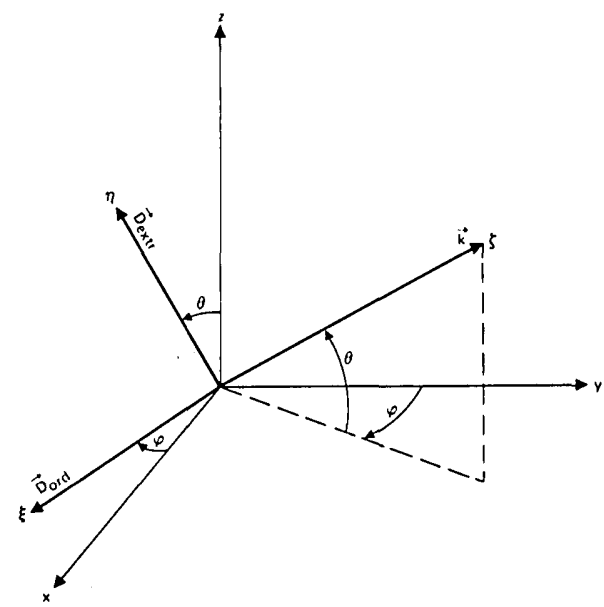

Fig. 1. Coordinate axis systems used to define crystal orientation and wave-propagation vector in optically active uniaxial crystal.

$$
\nabla \times \nabla \times \mathbf{E}==-\mu_{0} \frac{\partial^{2}}{\partial t^{2}}\left(\varepsilon_{0} \mathbf{E}+\mathbf{P}\right) .
$$

When $a \neq 0$, the field amplitudes depend on $\zeta$. This dependence is obtained by substituting Eq. (2) into Eq. (3) and by using Eq. (1) for the transverse polarization. The result is

$$
\begin{aligned}
& \frac{\mathrm{d} \mathscr{E}_{\xi}}{\mathrm{d} \zeta}=\frac{k_{0}^{2} a}{2 k_{\xi}} \mathscr{E}_{\eta} \exp \left[-i\left(k_{\xi}-k_{\eta}\right) \zeta\right], \\
& \frac{\mathrm{d} \mathscr{E}_{\eta}}{\mathrm{d} \zeta}=-\frac{k_{0}^{2} a}{2 k_{\eta}} \mathscr{E}_{\xi} \exp \left[i\left(k_{\xi}-k_{\eta}\right) \zeta\right], \quad k_{0}=\omega\left(\mu_{0} \varepsilon_{0}\right)^{1 / 2},
\end{aligned}
$$

where we assumed a slow variation, i.e.,

$$
\left|\frac{\mathrm{d}^{2} \mathscr{E}_{\xi, \eta}}{\mathrm{d} \zeta^{2}}\right| \ll\left|k^{2} \xi, \eta \mathscr{E}_{\xi, \eta}\right|
$$

The coupled set of equations can be symmetrized by defining

$$
E_{\eta} \equiv \mathscr{E}_{\eta} \sqrt{n_{\eta}}, \quad E_{\xi} \equiv \mathscr{E}_{\xi} \sqrt{n_{\xi}},
$$

which transforms Eq. (4) to

$$
\begin{aligned}
& \frac{\mathrm{d} E_{\xi}}{\mathrm{d} \zeta}=\kappa_{\xi \eta} E_{\eta} \exp \left[-i\left(k_{\xi}-k_{\eta}\right) \zeta\right] \\
& \frac{\mathrm{d} E_{\eta}}{\mathrm{d} \zeta}=-\kappa_{\xi \eta} E_{\xi} \exp \left[i\left(k_{\xi}-k_{\eta}\right) \zeta\right]
\end{aligned}
$$

where

$$
\kappa_{\xi \eta}=\frac{k_{0} a}{2\left(n_{\xi} n_{\eta}\right)^{1 / 2}} .
$$

The two eigenmodes of the medium can be obtained by a diagonalization $^{7}$ of Eq. (5),

$$
\begin{aligned}
& \mathbf{E}_{1}=\left|\begin{array}{c}
E_{\xi}^{\prime}(\zeta) \\
E^{\prime}{ }_{\eta}(\zeta)
\end{array}\right|=\left|\begin{array}{l}
E_{\xi}(\zeta) e^{-i k_{\xi} \zeta} \\
E_{\eta}(\zeta) e^{-i k_{\eta} \zeta}
\end{array}\right|=\left|\begin{array}{c}
i \kappa^{*} \\
\delta+\left(\kappa^{2}+\delta^{2}\right)^{1 / 2} \\
1
\end{array}\right| \exp \left\{-i\left[\mathbf{k}-\left(\kappa^{2}+\delta^{2}\right)^{1 / 2}\right] \zeta\right\} \\
& \mathbf{E}_{2}=\left|\begin{array}{l}
E_{\xi}^{\prime}(\zeta) \\
E^{\prime}{ }_{\eta}(\zeta)
\end{array}\right|_{2}=\left|\begin{array}{l}
E_{\xi}(\zeta) e^{-i k_{\xi} \zeta} \\
E_{\eta}(\zeta) e^{-i k}{ }_{\eta \zeta}
\end{array}\right|_{2}=\left|\frac{i \kappa^{*}}{\delta-\left(\kappa^{2}+\delta^{2}\right)^{1 / 2}}\right| \exp \left\{-i\left[\mathbf{k}+\left(\kappa^{2}+\delta^{2}\right)^{1 / 2}\right] \zeta\right\}
\end{aligned}
$$

i.e., they become circularly polarized. The coupling constant $\kappa$ depends on direction and can become small, or even zero. When $\kappa \gg \delta$, the eigenmodes approach

$$
\begin{aligned}
& \mathbf{E}_{1}=\left|\begin{array}{l}
0 \\
1
\end{array}\right| e^{-i k_{\xi} \zeta}, \\
& \mathbf{E}_{2}=\left|\begin{array}{l}
1 \\
0
\end{array}\right| e^{-i k_{\eta} \zeta},
\end{aligned}
$$

which are the linearly polarized eigenmodes of a birefringent crystal with no optical activity.

The problem of light propagation in an optically active crystal can be handled in one of two (equivalent) ways:

(1). In the first method, we resolve an arbitrary input field $\mathbf{E}(0)$ at the input $(\zeta=0)$ into the two eigenmode components $\mathbf{E}_{1}$ and $\mathbf{E}_{2}$ and propagate each one with its appropriate propagation constant, as in Eq. (7). The total field at any $\zeta$ is obtained by adding the two vectors. As an example:

$$
E_{\xi}(\zeta)=E_{\xi}^{\prime}(\zeta)_{1}+E_{\xi}^{\prime}(\zeta)_{2}
$$

(2) In the second method, we solve the coupled Eqs. (5) subject to the boundary conditions at $\zeta=0$. If, as an example, $E_{\xi}(0)=0$ and $E_{\eta}(0)=E_{0}$, then

$$
\begin{aligned}
E_{\xi}= & E_{0} \frac{\kappa_{\xi \eta} e^{-i \delta \zeta}}{\left(\kappa^{2}+\delta^{2}\right)^{1 / 2}} \sin \left[\left(\kappa^{2}+\delta^{2}\right)^{1 / 2}\right] \zeta, \\
E_{\eta}= & E_{0} e^{i \delta \zeta}\left\{\cos \left[\left(\kappa^{2}+\delta^{2}\right)^{1 / 2}\right] \zeta\right. \\
& \left.-i \frac{\delta}{\left(\kappa^{2}+\delta^{2}\right)^{1 / 2}} \sin \left[\left(\kappa^{2}+\delta^{2}\right)^{1 / 2}\right] \zeta\right\} .
\end{aligned}
$$

\section{OPTICAL ACTIVITY IN THE PRESENCE OF THE ELECTRO-OPTIC EFFECT}

Crystals that are optically active are often also electro-optic. The problem of light propagation in such crystals in the 
presence of dc or low-frequency electric fields is of practical interest. The solution also serves to illustrate the power of the coupled-mode formalism.

A dc electric field with components $E_{k}$ causes the elements of the dielectric tensor to change by ${ }^{7,8}$

$$
\Delta \varepsilon_{\xi \eta}=-\varepsilon_{0} n_{\xi}^{2} n_{\eta}^{2} r_{\xi \eta k} E_{k},
$$

where $r_{\xi \eta k}$ is the electro-optic tensor. Relation (1) now becomes

$$
\left|\begin{array}{l}
P_{\xi} \\
P_{\eta}
\end{array}\right|=\varepsilon_{0}\left|\begin{array}{lr}
n_{\xi}{ }^{2}-1 & i a-n_{\xi}{ }^{2} n_{\eta}{ }^{2} r_{\xi \eta k} E_{k} \\
-i a-n_{\xi}{ }^{2} n_{\eta}{ }^{2} r_{\xi \eta k} E_{k} & n_{\eta}{ }^{2}-1
\end{array}\right|\left|\begin{array}{l}
\mathscr{E}_{\xi} \\
\mathscr{E}_{\eta}
\end{array}\right|,
$$

where we have assumed that $\Delta \varepsilon_{i i}=0$.

From a comparison of Eq. (11) with Eq. (1), it follows that all our previous results apply, provided that we replace

$$
\kappa_{\xi \eta} \rightarrow \kappa+i \Gamma, \quad \kappa_{\eta \xi} \rightarrow-\kappa+i \Gamma,
$$

where

$$
\kappa=\frac{k_{0} a}{2\left(n_{\xi} n_{\eta}\right)^{1 / 2}}
$$

and

$$
\Gamma=1 / 2 k_{0}\left(n_{\xi} n_{\eta}\right)^{3 / 2} r_{\xi \eta k} E_{k} .
$$

This causes solution (10) for the field inside the crystals to become

$$
\begin{aligned}
E_{\xi}(\zeta)= & E_{0} \frac{(\kappa+i \Gamma) e^{-i \delta \zeta}}{\left(\kappa^{2}+\Gamma^{2}+\delta^{2}\right)^{1 / 2}} \sin \left[\left(\kappa^{2}+\Gamma^{2}+\delta^{2}\right)^{1 / 2}\right] \zeta \\
E_{\eta}(\zeta)= & E_{o} e^{i \delta \zeta}\left\{\cos \left[\left(\kappa^{2}+\Gamma^{2}+\delta^{2}\right)^{1 / 2}\right] \zeta\right. \\
& \left.-\frac{i \delta \sin \left[\left(\kappa^{2}+\Gamma^{2}+\delta^{2}\right)^{1 / 2}\right] \zeta}{\left(\kappa^{2}+\Gamma^{2}+\delta^{2}\right)^{1 / 2}}\right\}
\end{aligned}
$$

when the incident field is polarized only along $\eta$.

Equations (15) apply equally well in cases in which the index perturbation is due to strain or a sound wave. We need merely represent the effect of the perturbation as in Eq. (11) and from it deduce the appropriate form of $\Gamma$.

\section{COMPARISON OF THEORY AND EXPERIMENT WITH $\mathrm{AgGaS}_{2}$}

A particularly interesting and effective crystalline material that is useful for validating the above theory by experimental measurements is $\mathrm{AgGaS}_{2}$. This material (symmetry $\overline{4} 2 \mathrm{~m}$ ) is both birefringent and optically active. In addition, the birefringence goes to zero and changes sign at $497 \mathrm{~nm}$, near the band edge. A narrow-band electro-optic filter based on this property was recently proposed. ${ }^{9}$ The optical activity in $\mathrm{AgGaS}_{2}$ is strong, amounting to $522 \mathrm{deg} / \mathrm{mm}$ at the isotropic point, where the measurement ${ }^{10}$ is readily made, since the material exhibits pure circular birefringence there.

The coupling constant $\kappa$ is given by a tensorial relationship, ${ }^{6}$

$$
\kappa=\sum_{i j} \rho_{i j} l_{i} l_{j},
$$

where the $l_{i}, l_{j}$ are direction cosines of the wave-propagation vector with respect to the crystallographic principal axes. In $\mathrm{AgGaS}_{2}$,

$$
\rho_{11}=-\rho_{22}=\rho,
$$

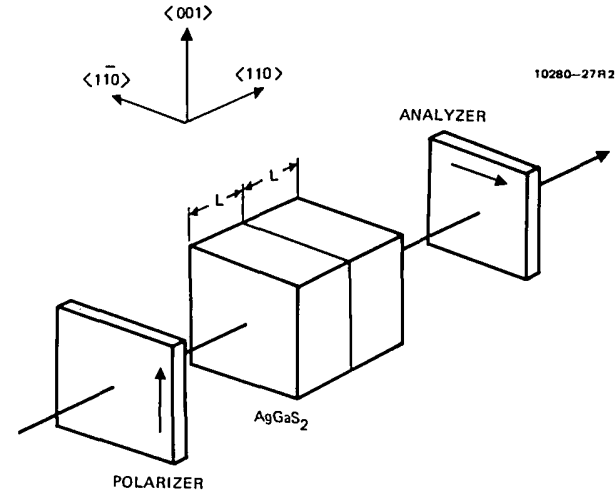

Fig. 2. Experimental arrangement of $\mathrm{AgGaS}_{2}$ crystal elements for measurement of spectral-transmittance characteristics.

and all other components are zero. Thus optical activity is absent for light rays that lie anywhere in the (110) or (110) planes.

With a configuration such as that shown in Fig. 2, for which the normal to the input surface is a long $\langle 110\rangle, \kappa$ varies as

$$
\kappa=\rho \cos ^{2} \theta \sin 2 \psi,
$$

where $\psi$ is the angle between the $\langle 110\rangle$ direction and the projection of the propagation vector on the (001) plane; that is, referring to Fig. $1, \psi=\phi-\pi / 4$. The birefringence parameter $\delta$ varies angularly in accordance with the following relationship:

$$
\begin{aligned}
\delta & =\frac{\pi}{\lambda}\left(n_{e}-n_{0}\right) \cos ^{3} \theta \cos \psi \\
& =\frac{\pi}{\lambda} C\left(\lambda_{0}-\lambda\right) \cos ^{3} \theta \cos \psi,
\end{aligned}
$$

where use has been made of the close approximation

$$
n_{\eta}-n_{\xi}=\left(n_{e}-n_{0}\right) \cos ^{2} \theta,
$$

$n_{e}-n_{0}$ being the (wavelength-dependent) full birefringence of the crystal, and where $C$ is the measured rate of change of birefringence with wavelength ${ }^{9-11}$ through the isoindex point $\lambda_{0}$.

In the analysis and computer programming of coupledmode processes, it is convenient to use a matrix operator formalism

$$
\left|\begin{array}{c}
E_{\xi}(\zeta) \\
E_{\eta}(\zeta)
\end{array}\right|=\left|\begin{array}{ll}
G_{11} & G_{12} \\
G_{21} & G_{22}
\end{array}\right|\left|\begin{array}{c}
E_{\xi}(0) \\
E_{\eta}(0)
\end{array}\right|
$$

for the evolution of the amplitudes of the principal polarizations (in the base representation of linearly polarized eigenmodes). The approach is particularly useful in describing the evolution of light through a succession of optical elements. This will become evident in what follows.

From the most general solution of Eqs. (5), in which both initial field amplitudes are nonzero, the following matrix elements of the $\mathbf{G}$ operator may be shown to result:

$$
\begin{aligned}
& G_{11}=\cos S \zeta+i\left(\frac{\delta}{S}\right) \sin S \zeta, \\
& G_{12}=\left(\frac{\kappa+i \Gamma}{S}\right) \sin S \zeta, \\
& G_{21}=\left(\frac{-\kappa+i \Gamma}{S}\right) \sin S \zeta, \\
& G_{22}=\cos S \zeta-i\left(\frac{\delta}{S}\right) \sin S \zeta,
\end{aligned}
$$



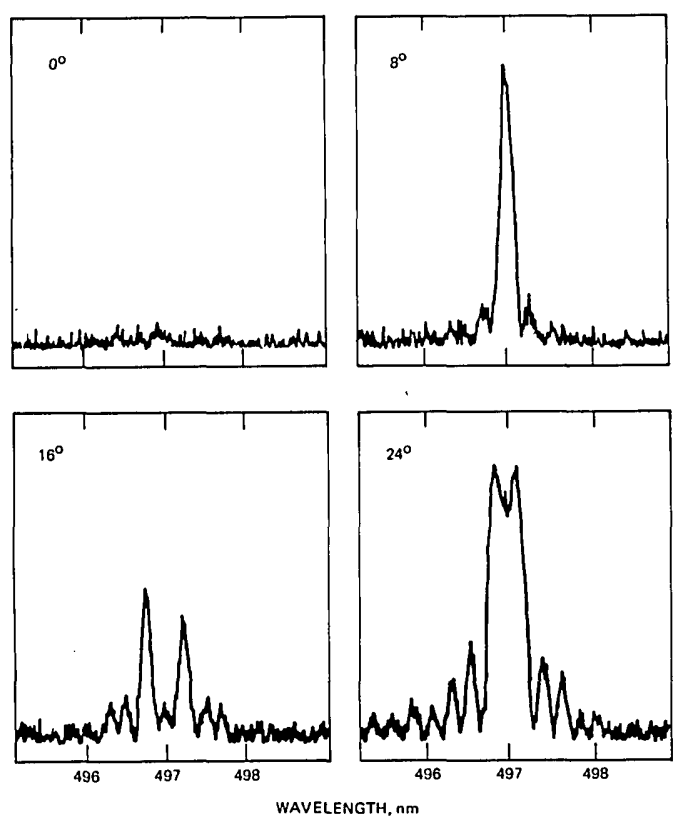

Fig. 3. Sample spectrophotometer traces of transmittance of a 1.48-mm sample of (110) $\mathrm{AgGaS}_{2}$ between crossed polarizers for collimated light at selected external angles of incidence in the (110) plane. (Configuration of Fig. 2).
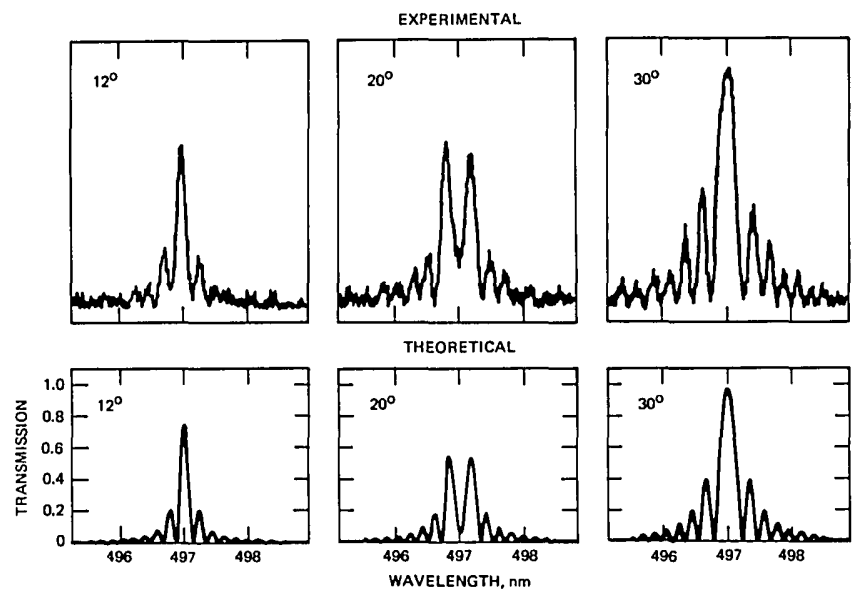

Fig. 4. Additional spectrophotometer traces as in Fig. 3, including corresponding computer simulations based on analytical model.

where

$$
S \equiv\left(\kappa^{2}+\Gamma^{2}+\delta^{2}\right)^{1 / 2}
$$

and where the electro-optic coupling constant $\Gamma$ is included for completeness. \{The phase factors $\exp ( \pm i \delta\})$ that appear in Eqs. (15), for example, have been included with those of the respective propagation constants $k_{\xi}$ and $k_{\eta}$ [see Eq. (2)] to yield a common propagation constant $\mathbf{k}$. We may thus omit such factors from Eqs. (21) and (22).\}

In order to establish the validity of our analytical model, we made detailed spectral-transmittance measurements on (110) samples of $\mathrm{AgGaS}_{2}$ located between crossed polarizers, using a well-collimated light beam from a white-light source at selected internal angles $\psi$ off normal incidence with the beam axis in the (001) plane, i.e., $\theta=0$. All measurements were made with no voltage applied to the crystal samples. ${ }^{9}$ With the input beam polarized along the optic axis, as shown in Fig. 2, the theory predicts that the relative transmittance $\tau$ through a crossed analyzer is [for $E_{\eta}(0)=1, E_{\xi}(0)=0, \Gamma=$ $0)$ ]

$$
\begin{aligned}
\tau & =\left|E_{\xi}(2 L)\right|^{2}=\left|G_{12}(2 L)\right|^{2} \\
& =\frac{\kappa^{2} \sin ^{2}\left[2 L\left(\kappa^{2}+\delta^{2}\right)^{1 / 2}\right]}{\kappa^{2}+\delta^{2}} .
\end{aligned}
$$

The spectrophotometric measurements were made in the neighborhood of $497 \mathrm{~nm}$ using two identical (110) plates of $\mathrm{AgGaS}_{2}$ of thickness $L=0.74 \mathrm{~mm}$. Measurements were first made by using both plates in tandem, with optic axes parallel, so that we effectively had a single plate of thickness $1.48 \mathrm{~mm}$. External angles of incidence were selected from 0 to $30^{\circ}$. Figure 3 shows the results for four representative cases: 0 , 8,16 , and $24^{\circ}$. It is apparent from these spectral transmittances that the optical activity engenders significant coupling of polarization components in the neighborhood of the isoindex point at incidence angles other than zero. Figure 4 shows additional spectrometer traces for external incidence angles of 12,20 , and $30^{\circ}$. Appearing with each is the corresponding computer plot based on Eq. (24), together with Eqs. (18) and (19), and the following values of the constants:

$$
\begin{aligned}
\lambda_{0} & =0.497 \mu \mathrm{m}, \\
\rho & =9.1 \times 10^{-3} \mathrm{rad} / \mu \mathrm{m}\left(=522^{\circ} \mathrm{mm}^{-1}\right), \\
L & =740 \mu \mathrm{m}, \\
C & =1.87 \mu \mathrm{m}^{-1} .
\end{aligned}
$$

It is apparent from the agreement of these representative computer simulations that the analytical model is valid.

As a further test of the theory, we measured the spectral characteristics of the crystal pair with the second element rotated through $180^{\circ}$ about the beam axis (the $\langle 110\rangle$ direc-
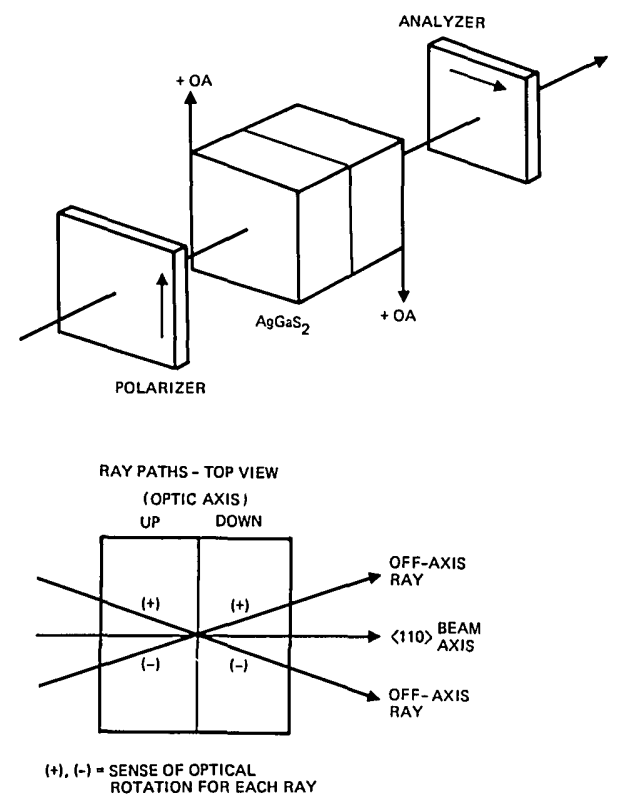

Fig. 5. Two-element antiparallel configuration for illustrating cancellation of optical coupling through optical activity in $\mathrm{AgGaS}_{2}$ at the isoindex wavelength. 


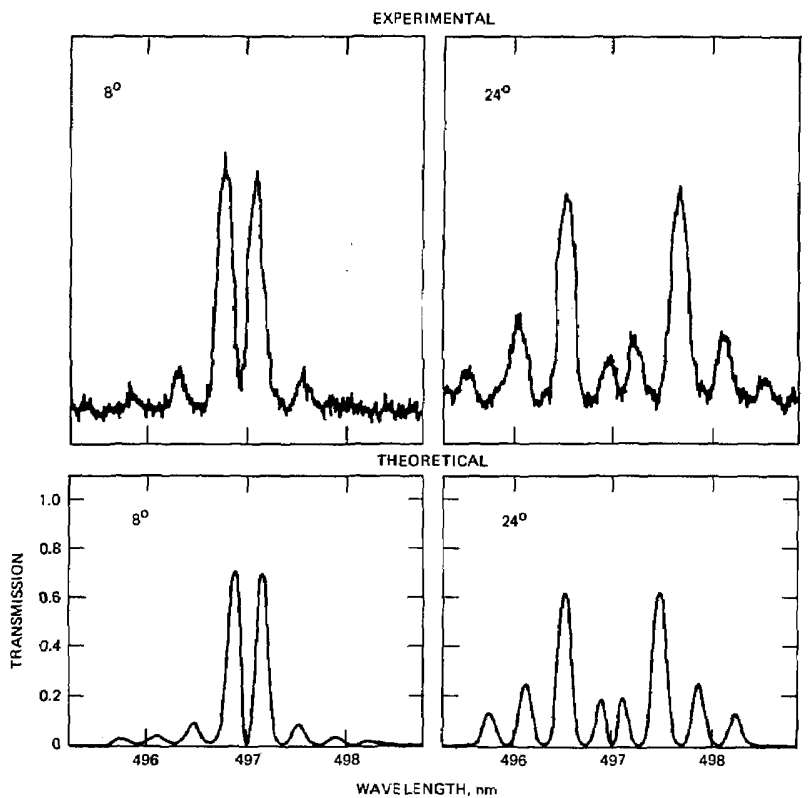

Fig. 6. Examples of spectrophotometer traces for the two-element antiparallel configuration of Fig. 5, together with corresponding computer simulations. Angles shown are external angles of incidence.

tion) so that the positive optic axes of the two crystal halves were antiparallel, as is shown in Fig. 5. With this arrangement, off-normal rays at the isoindex wavelength should experience rotatory powers of opposite sense in the two crystal elements [see Eqs. (17) and (18)], thereby unwinding the effect of optical activity since at this wavelength pure circular birefringence occurs; that is, the eigenmodes are right and left circularly polarized. At wavelengths away from this point, such is no longer the case-the eigenmodes being elliptically polarized-and coupling does indeed occur. The effect is illustrated in Fig. 6 by two examples of experimental spectrometer traces, and accompanying computer simulations, for the cases of external incidence angles 8 and $24^{\circ}$. Again, excellent agreement between experimental results and the computer model is noted. It should be further noted that the transmittance at all angles of incidence is indeed zero at the isoindex wavelength.

In formulating the analytical result, an effective matrix operator

$$
\boldsymbol{g}=\mathbf{G}(-\kappa, L) \cdot \mathbf{G}(\kappa, L)
$$

was used, namely, a product of two $G$ operators, in which the sign of $k$ in the second was opposite that in the first, in accordance with the experimental arrangement of the two crystals elements of Fig. 5.

\section{CONCLUDING REMARKS}

The techniques of coupled-mode theory, so widely used and extensively exploited in such diverse disciplines as integrated optics (modulators, mode converters, filters) and quantum mechanics (dipole transition probabilities), have here provided further clarification and physical insight into the nature of light propagation in optically active, birefringent crystals. It is hoped that the explicit solutions derived in this paper for the power transfer between principal polarizations may help to resolve any elements of mystery and uncertainty characterizing earlier theories. ${ }^{6}$

\section{ACKNOWLEDGMENTS}

The technical assistance of D. P. Devor, J. G. Johnston, R. R. Stephens, and C. M. Walker is gratefully acknowledged.

This research was supported in part by the U.S. Office of Naval Research.

\section{REFERENCES}

1. G. Szivessy, "Kristaloptik," in Handbuch der Physik, H. Geiger and K. Sheel, eds. (Springer-Verlag, Berlin, 1928), Vol. 22, pp. 635-904.

2. M. Born, Optik (Springer-Verlag, Berlin, 1933), pp. 413-420.

3. E. U. Condon, "Theories of optical rotary power," Rev. Mod. Phys. 9, 432-457 (1937).

4. G. N. Ramachandran and S. Ramaseshan, "Crystal optics," in Handbuch der Physik, S. Flügge, ed. (Springer-Verlag, Berlin, 1961), Vol. 25, No. 1, pp. 76-85, 97-106.

5. J. P. Mathieu, "Activité optique naturelle," in Handbuch der Physik, S. Flügge, ed. (Springer-Verlag, Berlin, 1961), Vol. 28 , pp. 333-431.

6. J. F. Nye, Physical Properties of Crystals (Clarendon, Oxford, 1976), Chap. 14.

7. A. Yariv, "Coupled-mode theory for guided wave optics," IEEE J. Quantum Electron. QE-9, 919-933 (1973).

8. I. P. Kaminow, An Introduction to Electrooptic Devices (Academic, New York, 1974), p. 56.

9. J. F. Lotspeich, "Iso-index coupled-wave electrooptic filter," IEEE J. Quantum Electron. QE-15, 904-907 (1979).

10. M. V. Hobden, "Optical activity in a non-enantiomorphous crystal: AgGaS 2 ," Acta Crystallogr. Sect. A 24, 676-680 (1968).

11. G. D. Boyd, H. Kasper, and J. H. McFee, "Linear and nonlinear optical properties of $\mathrm{AgGaS}_{2}, \mathrm{CuGaS}_{2}$, and $\mathrm{CuInS}_{2}$, and theory of the wedge technique for the measurement of nonlinear coef ficients," IEEE J. Quantum Electron. QE-7, 563-573 (1971). 